\title{
ESTUDIOS
}

\section{Organizaciones institucionales de Europa Occidental}

\author{
Por Alfonso ECHANOVE :
}

\section{Alcance de este artículo}

La tendencia de Europa hacia su unificación, tomando de este proceso exclusivamente el tramo recorrido desde el final de la Segunda Guerra Mundial, aparece hoy a nuestros ojos con una frondosidad tal vez excesiva. Frondosidad que se manifiesta en la multiplicidad y variedad de organizaciones que reclaman para sí en diverso grado una finalidad unificadora del continente. Esta frondosidad no sería difícil de explicar o discernir si uno solo fuera el tronco común que alimenta y da vida a todas estas organizaciones. Pero esta atribución es más que discutible.

Europa vive políticamente inserta, nación por nación, en un conjunto de organizaciones internacionales más amplio que ella misma. En tal sentido puede decirse que de alguna manera la Organización de las Naciones Unidas es también una organización de Europa (o algo en virtud de lo cual Europa se ve algo organizada), en cuanto que los países europeos en ella integrados se someten a unas determinadas reglas unificadoras. Lo mismo puede decirse de la Organización Defensiva del Atlántico Norte (NATO).

Las organizaciones que, sin embargo, no dudaríamos en llamar eutopeas son muy numerosas, de muy distinta finalidad, y de muy distinto alcance geográfico-político. Así, por ejemplo, existen organizaciones europeas que cubren toda la Europa Occidental en un intento unificador, cual es la O.C.D.E. (transformación de la antigua O.E.C.E.); o, asimismo, una serie de organizaciones técnicas en los más diversos campos. Pero al lado de éstas coexisten organizaciones europeas de ámbito parcial $\mathrm{y}$, sin embargo, de primer orden, como son todas las integradas en lo que se llama ya Comunidades Europeas, bien que cubran solamente a seis, nueve o doce países, si la última ampliación se realiza. Lo mismo se diga de la E.F.T.A.

\footnotetext{
* Doctor en Historia.
} 


\section{A. ECHANOVE}

No termina ahí la dificultad de coaligar estos organismos en una inspiración común. Algunas de estas organizaciones son de carácter eninentemente polinico-defensivo, como la Unión Europea Occidental, y otras, en las que prevalece el interés político-juridico, como el Consejo de Europa, o el económico, como la C.E.E. ¿Qué lazos unen a todas estas otganizaciones? ¿Cuál de ellas es más europea? ¿Son necesarias tantas organizaciones, aun de matices diferentes, para llegar a la finalidad úlima de la unificación europea? ¿Cuál de ellas, si es que hay alguna, puede conseguir llegar la primera a la meta en esta competición pos dat organicidad supranacional a nuestro continente? ¿Dónde empieza Europa y dónde ter. mina, para saber cuál es el alcance y el tipo de otganización que permitiria coordinar tantos intereses diversos y encontrados como en Europa se mueven?

Estas preguntas, que nos sumergen en gran perplejidad, no se pueden contestar de un modo claro y unívoco. En las líneas que siguen vamos a tratar de aislar aquellas organizaciones europeas que nos parecen más importantes o medulares, describiéndolas sobre todo a partir de su origen, para que a partir de ellas se pueda juzgar el alcance que actualmente tienen en la construcción europea.

\section{Génesis del actual movimiento europeista}

No tomamos el agua desde muy arriba, porque entrat en el origen más profundo de las tendencias unificadoras europeas es pretender describir casi una filosofía de la historia de este maltratado y en otro tiempo poderoso continente. Los movimientos europeos propiamente tales que han conseguido por primera vez plasmarse en organizaciones tienen su origen en la conciencia común de la derrota experimentada al final de la Segunda Guerra Mundial. Todos los países europeos-y en este sentido hay que excluir a Rusia, como paralelamente a los Estados Unidos, que fueton los vencedores reales de la contienda-, se han sentido esencialmente derrotados en 1945 cualesquiera hayan sido su alineación, su régimen político o sa adscripción ideológica. A este hecho fundamental ha venido a sumarse la división establecida por los vencedores en la conferencia de Potsdam, en virtud de la cual, lo que acostumbrábamos a llamar Europa se vio escindido en 1945 por un eje Norte-Sur que incluso desmembraba a una de las grandes potencias europeas.

Este clima de derrota se vio acentuado además por dos matices contrapuestos: por un lado se establecía una Organización mundial de las Naciones Unidas, en la que Europa como tal no tenía significación propia, sino en tanto en cuanto sus diversas naciones quedaban en ella integradas y no del todo, por la ausencia muy cualificada de Alemania y de algunos otros países, entre ellos el nuestro, por muy variadas razones. El segundo matiz se puso de manifiesto ahondando la división europera desde el momento en que se vio que lejos de provocar un acto colectivo de contrición por la desunión de las décadas precedentes, la guerra parecía haber resucitado en cada uno de los países europeos más importantes un nacionalismo egoísta difícil de compaginar con cualquier movimiento unificador. 
Sin embatgo, precisamente en este clima de absoluto desconcierto, algunas minorias, entre esperanzadas e ilusas, intuyen que el futzro de Europa sólo puede estar en una integración.

Estas minorías, que continuando el símil de la formación vegetal vendríar a constituir como las raíces de las que luego surgiría un movimiento europeo, fueron por la segunda mitad de la década de los cuarenta, justo al final de la guerra mundial, mucho más numerosas y pujantes de lo que puede creerse. Sus componentes consideraban, con un poco de precipitación sin duda, que el movimiento unionista o federalista había alcanzado en Europa la edad adulta. Al menos no se podía decir que fuera despreciable. He aquí algunos datos.

Existían en el año 1946 hasta $45^{\circ}$ asociaciones federalistas, muchas de las cuales estaban vinculadas entre sí como miembros de una Unión Europea de Federalistas, que había sido creada en Basilea y París en diciembre del mismo año. Presidente de esta organización era Henri Brugmans, bien conocido animador europeísta belga, más tarde director del Colegio de Europa en Brujas.

Por países, el número era muy variado. En cabeza Francia con no menos de diez, seguida por Suiza con siete y Italia con seis; hasta cinco se contaban en Inglaterra y otras tantas en Bélgica, y no menor número Alemania, Austria, Dinamarca, Luxemburgo, Noruega, Holanda y Suecia. E incluso compartían este mismo espíritu, aunque desde fuera del continente, algunas asociaciones americanas que ya entonces empezaban a hacer campaña por un movimiento federalista mundial. Un buen número de publicaciones, unas 10 ó 12 , daban reflejo escrito a tantas conversaciones esperanzadas de club, como pueden deducirse de toda esta floración.

Estas asociaciones actuaron como grano de trigo enterrado en la arena. A partir del año 48 iban a ir extinguiéndose a medida que emergían en el panorama político las primeras realizaciones de repercusión internacional.

\section{1948: Año crucial para la construcción europea}

Puede considerarse el año 1948 como el año más importante en orden a la construcción europea. Por tres motivos coincidentes: el primero, porque el 17 de marzo se constituyó, mediante el Tratado de Bruselas, un principio de organización defensiva que habría de ser el germen de las sucesivas uniones del mismo género hasta la actualidad. En segundo lugar, porque en el mes de mayo, el Congreso de La Haya, organizado por diversos movimientos federalistas europeos de los anteriormente indicados, que se habían reunido en un organismo especial (Comité Internacional de Coordinación de los Movimientos pro Unión Europea, C.I.M.U.E.), votó una resolución recomendando la formación de una Asamblea Europea.

Mediante ambos acontecimientos quedaron en principio englobadas $1 a$ rendencia unificadora de defensa, que en aquellos años se consideraba 


\section{A. ECHANOVE}

no sin motivo absolutamente prioritaria, y toda la materia de consideración política que juntamente con el organismo défensivo y apoyada en é aspiraba a desarrollar todo el movimiento general de envergadura y finalidad ilimitados. En tercer lugar, por si poco fuera, y con el beneplácito de los Estados Unidos, nació aquel mismo año una organización cuya finalidad inmediata consistia en canalizar las ayudas norteamericanas del plan Mashal, la Organización Europea de Cooperación Económica (O.E.C.E.), que aglutinó los intereses especificamente económicos.

E1 18 de agosto se verificó de hecho el ensamblaje organizativo de las dos tendencias principales, la militar y la política, cuando el mismo movimiento (C.I.M.U.E.), que habia promovido el Congreso de La Haya, presentó al Tratado de Bruselas un memorándum en que oficialmente formulaba la susodicha aspiración a una Asamblea política europea. De este memorándum habría de nacer el Consejo de Europa.

El que desee seguir ordenadamente las vicisitudes de los movimientos europeos habrá, por tanto, de establecer las siguientes prioridades:

a) Movimientos relacionados con la defensa militar de Europa como continente.

b) Movimientos de organización política de Europa, apoyados en la defensa.

c) Organizaciones de cooperación o integración económica.

No hay arbitrariedad en este orden. La unidad europea comenzó de hecho en un momento históricamente difícil, por el acuerdo político-militar de las principales potencias a partir del Tratado de Bruselas o Unión Europea (y alentado por los Estados Unidos). Inmediatamente, las iniciativas que habían conseguido plasmar un cierto proyecto político se dirigieron al organismo de defensa, el más sólido que en ese momento aglutinaba las políticas de las naciones europeas, para pedirle que apadrinara la coordi. nación política. ¿A quién si no? Al hacerlo así, mediante la creación del Consejo de Europa, los países miembros del Tratado de Bruselas creaban una nueva línea de desarrollo autónomo, que teóricamente debería proceder de un modo independiente, ya veremos con qué vicisitudes. Por último, y en ese mismo año, los centros de decisión nacionales que respaldaban a la organización defensiva (y subordinadamente a la política) crearon, por ciertas razones prácticas de emergencia, la línea de cooperación económica con el organismo inicial de la O.E.C.E. 


\section{DESARROLLO DE LA LINEA DEFENSIVO-MULITAR}

Resumen: Comprende, a) la Unión Oecidental de 1948; b) el Tratado del Atlático Norte; c) la racasada Comum nidad turopea de Defensa, en 1954, y d) la Unión curopea Decidental, asimismo en 1954, en sustitución de la non nata C.E.

\section{El Tratado de Bruselas de 1948}

Puede considerarse como la primera concreción de un movimiento europeo relativamente generalizado.

Esencialmente fue una alianza defensiva establecida para cincuenta años y firmada en Bruselas el 17 de marzo de 1948 entre Francia, Gran Bretaña y los países del Benelux. Este tratado, concluido en el marco de la organización militar de Europa occidental, instituyó un Comité de Defensa que reunía a los ministros de Defensa de los estados miembros, un Comité militar permanente con sede en Londres y un Estado Mayor de las fuerzas armadas de Europa occidental, instalado en Fontainebleau, bajo la dirección de los generales Montgomery y De Lattre. Este Tratado es, en su espíritu y en su forma, el verdadero precursor del Atlántico Norte. Sus organismos se fundieron en 1950-51 en los de la NATO. Los textos del Tratado de Bruselas fueron, sin embargo, reasumidos, tras el fracaso de la Comunidad Europea de Defensa, en agosto de 1954, para servir de base a la Unión de Europa Occidental, creada por los Acuerdos de Paŕs en ese año.

\section{Tratado del Atlántico Norte}

Por extraño que parezca, esta organización, en la que la presencia de países no europeos y, sobre todo, de los Estados Unidos, parece que la hacen desbordar el marco previsto en estas líneas, es la que tiene un origen más hondamente europeo.

La Organización del Tratado del Atlántico Norte (O.T.A.N. o N.A.T.O., según su formulación francesa o inglesa respectivamente) se constituyó el 4 de abril de 1949 en Washington, como consecuencia de la presión de los cinco paises de la Unión Occidental o Pacto de Bruselas, descrito en el párrafo anterior. Es una organización nacida de la angustia europea. Mediante ella, los Estados Unidos, aceptan la coalición con los Estados de Europa, admitiendo su colaboración conjunta, que consolida su estrategia en la zona más vital y amenazada, pero adquiriendo un protagonismo dominante de doble efecto, positivo y negativo, sobre los países protegidos.

El hecho de que además de la superpotencia americana se integren en este Tratado el Canadá, Italia, Dinamarca, Islandia y Noruega; más tarde Grecia, Turquía y la R.F.A., disminuye correlativamente la condición pre- 


\section{A. ECHANOVE}

ferente de cualquiera de los países integrantes (léase Inglaterra o Francia), y desplaza hacia los Estados Unidos el centro de gravedad.

En la medida en que esta obligada enajenación de responsabilidades se realiza, se hipotecarán todas las organizaciones europeas posteriores hasta el día de hoy. Sólo el proyecto de Kennedy de promover una unificación europea que hiciera a ntesro continente un partenaire en igualdad de condiciones con los Estados Unidos hubiera podido permitir el levantamiento de esta hipoteca básica. Pero la muerte prematura del presidente acabó también en raiz con este sueño.

La N.A.T.O. u O.T.A.N. fue, es y será, por tanto, el único escudo viable para Europa, y garantía de seguridad para cualquier construcción europea. La travesura de Francia al pretender una emancipación de los Estados Unidos, tiene, pues, sólo un valor de gesto.

\section{La fracasada Comunidad Europea de Defensa (C.E.D.)}

Para comprender el por qué de la aparición de esta abortada unión occidental debemos situarnos en la alarma que creó la guerra de Corea en 1950. Este acontecimiento, de extremada gravedad en los mismos albores de la paz, sirvió para abrir los ojos a Occidente de una manera definitiva sobre dónde había que situar el verdadero y crucial antagonismo de los bloques. Europa derrotada seguía siendo al mismo tiempo el flanco más débil pero más necesario de la defensa occidental. En este contexto se empezó a alumbrar-en el seno de la Alianza Atlántica-ma idea de una defensa común compartida e incluso federada de los países europeos contra un enemigo común que superaba con mucho al remoto peligro alemán. Más aún, debía servir para aventar muchos recelos antialemanes y desear más bien la participación alemana en el mismo proyecto.

La idea ya había nacido en el Congreso de La Haya de 1948 que dio lugar a otras iniciativas europeísticas. En 1951 fue ya claramente formulada por el primer ministro francés René Pleven, pero quien la trató de poner en práctica en 1954-y casi lo consiguió--fue el gran europeísta Robert Schumann, a instancias de Jean Monnet, al que se conoce como «padre de Europa».

Esencialmente, el proyecto consistía en formar una fuerza militar unitaria e integrada por los países europeos, que tuviera el doble efecto de neutralizar la posibilidad alemana de rearme incluyendo a esta nación en la cooperación defensiva, y por otra, creara una fuerza supranacional verdaderamente eficaz. En la mente de Monnet y de Schumann, este proyecto iba unido a otros desarrollos de tipo económico, guiados por la misma intención, que condujeron en ese mismo año a la creación de la Comunidad Europea del Carbón y del Acero (C.E.C.A.).

Ante el proyecto, los estados participantes en la N.A.T.O. manifestaron diversas posturas. En general, los países pequeños y débiles se mos. 
traron favorables. En Francia, la opinión se dividió, por incluir el rearme alemán tanto como por implantat una supranacionalidad que conllevaba pérdida de soberanía. Por lo que respecta a Inglaterra, estaba menos dispuesta que nadie a la idea de la suptanacionalidad miltar, pero no deseaba absolutamene que la organzación defensiva desapareciera. Unicamente rehusaba el precio político que le conrespondua. Esta ambigua postura explica su posterior reacción, así como también la francesa.

Todo este planteamiento fracasó estrepitosá y sensacionalmente cuando el 30 de agosto de 1954 la Asamblea Nacional francesa se negó a ratificar el proyecto. Por una parte había prevalecido el recelo secular hacia o contra Alemania. Por otra, empezaba a pujar con fuerza el neonacionalismo del genetal De Gaulle que aspiraba a que cualquier tipo de alianza defensiva común se hiciera sobre la base de Estados absolutamente soberanos, suficientemente coordinados, y presididos o encabezados por el liderazo de Francia, que así mismo habría de ostentar la jefatura del SACEUR (Supreme Allied Commander Europe). Sólo así podría Trancia controlar el rearme alemán.

Sin embargo, y aunque la actitud de Inglaterra, como hemos dicho, no difería excesivamente de la gaullista en cuanto a la soberanía, el gobierno inglés se sintió fuertemente alarmado por el fracaso. Esto fue lo gue ocasionó una reacción de tesorte, muy ágil, dirigida por la imaginación política de Anthony Eden, por la que los gobiernos se pusieron febrilmente a elaborar una nueva modalidad de la unión defensiva europea. Querer es poder. Al cabo de escasamente dos meses estaba reformulado el proyecto de una Unión Europea Occidental que con las limitaciones que enseguida explicaremos, venía a llenar la laguna creada por la nacional francesa.

\section{La Unión Europea Occidental}

Esta asociación, de carácter defensivo militar, ha permanecido hasta el dia de hoy desde el momento de su institución en octubre de 1954, con el conjunto de textos llamados «Acuerdos de París». Fue, como queda dicho, el resultado del fracaso de la C.E.D., que actuó como estimulante, y sólo en el plazo de dos meses se consiguió una unión que, aunque era menos ambiciosa que la C.E.D., resultó estable y de alguna manera, también suficientemente operativa.

El objetivo de la Unión Europea Occidental, que no es una asociación de carácter excesivamente activo, está sobre todo en la fórmula de limitación y control de armamentos de los diversos países que la componen. De esta manera se consiguen simultáneamente finalidades que parecían contrapuestas y aun antagónicas. El control de armamentos facilita la vigilancia del rearme alemán, fantasma que permaneció vivo, comprensible. thente, desde el final de la Segunda Guerra Mundial, hasta que la aparición del peligro soviético lo relegó a segundo plano. Junto con eso, las operaciones de control de armamentos de los Estados miembros suponen 


\section{A. ECHANOVE}

en realidad una verdadera coordinación militar de los mismos, Alemania Federal incluida, con vistas a una posible defensa común. Así, se determina el nivel de «stocks» armamentísticos que cada Estado puede o debe tener, se prevé el estacionamiento de tropas de unos países en otros, concretamente en Alemania, y sobre todo, la organización sirve para facilitar un constante intercambio de puntos de vista de los jefes militares, lo cual facilita incuestionablemente la adopción de otros tipos de medidas de carácter político o económico, llegado el caso.

Actualmente la U.E.O. cuenta con siete Estados afiliados: los cinco miembros de la anterior Unión Occidental o Tratado de Bruselas, de que ya hicimos mención, a saber, Gran Bretaña, Francia y los tres del Benelux, más la República Federal Alemana e Italia.

Evidentemente, el radio de acción de la U.E.O. queda así fuertemente constreñido a unas operaciones de catácter casi más técnico-militar que verdaderamente político dentro de este cuadro de países. Porque estos mismos países, integrados en el marco más general de la N.A.T.O., se ven más condicionados por las necesidades estratégicas generales y las considetaciones de todo tipo que esta máxima organización, controlada por los Estados Unidos, impone a la estrategia global de Occidente. Hay que hacer la salvedad de que, así como Francia es miembro a todos los efectos de la U.E.O., respecto de la N.A.T.O. fue apartada de la organización por el general De Gaulle, aunque permaneciendo unida al principio esen. cial del Tratado, fómula bastante extraña y singular, que, sin embargo, no altera la eficacia sustancial de una u otra organización, estrechamente complementarias.

\section{DESARROLLO DE LA LINEA POLITICA}

Resumen: Prácticamente toda la línea política queda englobada en el Consejo de Europa, que cubre todo el pertodo, desde su fundación en 1949 hasta la actualidad. Su mayor realización es la Convención de los Derechos del Hombre en Europa.

\section{Consejo de Europa}

Fue fundado en mayo de 1949 para conseguir mayor unidad entre sus miembros, facilitar su progreso económico y social, y defender los principios de la democracia parlamentaria.

Aunque originariamente participaron en él diez países, actualmente cuentan hasta veinte con la incorpotación de España, último de los miembros adheridos. No se excluye por principio a los países del telón de acero, sino primordialmente porque estos Estados en ningún caso setían autorizados para ello por la U.R.S.S., aunque hipotéticamente lo desearan. Es el caso de Finlandia, que en un principio, aun reuniendo todos los requisitos pedidos por el Estatuto, no se atrevió a solicitar su entrada. 
También al principto quedaron fuera del Consejo, Suiza, Portugal y España, más tarde integradas. El caso de Alemania mereció amplias discusiones y al final se aprobó su entrada como miembro, condicionada a la entrada del entonces territorio autónomo del Sarte. A partir de 1957, este territorio pasó definitivamente a format parte de la R.F.A. como el dé cimo Lond, y el problema desapareció.

La organización de este Consejo se estructura de la siguiente forma:

a) Existe un Comité de Ministros, compuesto por los Ministros de Asuntos Exteriores de cada uno de los Estados, que decide sobre todas las materias de organización interna, hace recomendaciones a los gobiernos e incluso puede estructurar convenciones y acuerdos, todo ello con efecto vinculante. Se reúne dos veces al año, ordinariamente. Sin embargo, existe un Comité de delegados de los Ministros, que trabajan la mayor parte de los asuntos de rutina mediante reuniones mensuales, y sus decisiones tienen la misma fuerza que las adoptadas por los Ministros.

\section{b) La Asamblea Parlamentaria}

Se compone de 154 miembros, elegidos con un criterio proporcional a la magnitud demográfica del país correspondiente, pero favoreciendo especialmente a los países pequeños. Los miembros son elegidos por sus parlamentos nacionales o señalados por ellos. Se da en esta Asamblea Parlamentaria también la circunstancia de que todos los miembros son así mismo miembros de sus parlamentos, y los partidos representados por cada delegación guardan así mismo la proporcionalidad que poseen en sus propios parlamentos. Esta Asamblea Parlamentaria se reune en sesion orinaria una vez al año durante un tiempo que no debe exceder de un mes, si bien fraccionado en tres partes distintas (enero-febrero, abril-mayo y septiembre-octubre). Su función consiste en someter al Comité de Ministros roda clase de recomendaciones sobre múltiples materias y en estudiat y discutir cualesquiera materias que se consideren de común interés eutopeo. Como es lógico, la Asamblea se mantiene en una actividad constante a través de un Comité Permanente que se reúne tres veces al año. Dentro de la Asamblea existen, como en toda asamblea parlamentaria, una serie de comisiones que abarcan todos los campos usuales de competencia legislativa en los parlamentos nacionales.

\section{c) El Comité Mixto}

Está formado a base de la Comisión permanente, pero con mucha mayor autoridad, lo que hubiera sido, de haberse realizado con plenitud, una especie de embrión de gobierno europeo. Pero Inglaterra era hostil a la idea de este tipo de gobierno. De ahí que se rechazara el nombre de Comité Ejecutivo, y se constituyera un Comité Consultivo Mixto que asegutase el enlace entre los dos órganos del Consejo de Europa, y que está 


\section{A. ECHANOVE}

compuesto-bajo la presidencia del Presidente de la Asamblea-por siete miembros de ésta y cinco como mínino, del Comité de Ministros.

La sede del Consejo de Europa se halla en Estrasburgo y suele tener una sesión conjunta con su homólogo, el Parlamento de las Comunidades Europeas, del que le separan diferencias obvias de composición y finalidad legislativa, pues no tienen más soberania que la que reciben de los Estados miembros (los cuales no la delegan jamás) y que sólo se hace efectiva mediante la unanimidad de decisión y ratificación nacional.

Durante los primeros años, el Consejo de Europa llevó una vida progresivamente paralizada por las dos concepciones opuestas, la continental francomblga, de tendencia federalista, y la inglesa, de carácter meramente funcional.

Sin embargo, la fundación de la Comunidad Europea del Carbón y del Acero (18 de abril de 1951) formada por los seis países que luego habrian de ser los Seis del Mercado Común, tuvo la virtud de revitalizar los proyectos europeos, puesto que la Gran Bretaña empezaba a comprender que su idea de Europa le dejaba a la zaga de un movimiento más generalizado.

Este empuje vigoroso de la C.E.C.A. encontró opiniones contradictom rias entre los miembros del Consejo de Europa. Por una parte era un motivo de satisfacción para todos los partidarios de la unión europea el que por fin se hubiera encontrado un camino para comenzar la construcción sobre una base sólida. Pero era al mismo tiempo causa de preocupación y hasta de despecho el que todo esto se hubiera hecho al margen del Consejo, que durante los tres años anteriores había estado dedicado a esta única y exclusiva tarea.

A partix de entonces, y hasta el pasado año de 1978, el Consejo de Europa ha mostrado gran actividad, sacando adelante una suma de convenciones y pactos referentes a las más variadas materias en número de unos 70, casi todos ellos ratificados por los parlamentos nacionales, y por lo tanto, en vigor. La variedad e interés de estos convenios, si se toman globalmente y no por unidades a veces irrelevantes, explica por qué para algunos países europeos, sobre todo los más reticentes a más sólidas y autónomas uniones, este Consejo de Europa es la única institución verdaderamente europea que tiene porvenir, y la única de la que puede ir saliendo una Europa del futuro como ente autónomo.

La obra del Consejo de Europa es apreciada diversamente. Para los partidarios de una mera cooperación europea y no de una integración, el Consejo de Europa es un organismo casi perfecto. Su concepción y competencias globales, su adaptabilidad paciente, su ritmo obligadamente lento, gue parece hecho a la medida que tequieren los cambios de la mentalidad colectiva, su articulación legal suptanacional, llena de excepciones, matices y permisividades, hacen de é el verdadero embrión de an órgano legis- 
lativo tan complexivo como lo necesitaría una Europa total quizás uniticada en el futuro.

Para los europeístas más avanzados, en cambio, y en general para las Comunidades Europeas, que progresan más hacia la integración, el Consejo de Europa apenas es otra cosa que una apreciable caja de resonancia, un foro abierto de discusión y recomendaciones resultantes a los Estados miembros y un fautor de pequeños acuerdos pactados por para convención.

Para otros, en fin, por mera ignorancia de la labor jurídica que el Consejo está llevando a cabo, el Consejo de Europa es irrelevante en conparación con la Europa de las Comunidades, basada en la integración económica. «Más vale pájaro en mano...», parecen pensar.

\section{La Convención de Derechos del Hombre}

Pero cualquiera que sea el valor que se atribuye a la labor de conjunto del. Consejo, reflejada en tantos convenios y pactos de diversa monta, hay que hacer una inevitable y extremadamente positiva excepción. Lo que se considera como la obra maestra del Consejo de Europa desde su fundación es la Convención de los Derechos del Hombre, firmada el 4 de noviembre de 1950 .

Una mentalidad jurídica debe sentirse satisfecha con este gran factor de aglutinación ideológica, lleno de particularidades. En un artículo tan general y descriptivo como el presente, esta Convención no puede ser adecuadamente explicada. Sin embargo, no nos resistimos a señalar que se diferencia considerablemente de la Declaración de los Derechos Humanos proclamada por las Naciones Unidas en 1948. Por dos razones.

En primer término, porque al definirse el Consejo de Europa por una ideología política basada en el imperio de la ley y en el respeto de los valores individuales como patrimonio común europeo, contagia a la Convención de 1950 una determinada ética y consiguientemente, una determinada concepción de los Derechos del Hombre, mucho más precisa y orientada que pueda serlo la proclamada con carácter quizá excesivamente general por las Naciones Unidas.

En segundo lugar, porque dentro de la carencia de poder soberano de que el Consejo de Europa adolece, ha conseguido con gran habilidad hacer aceptables por sus miembros ciertos mecanismos de control y sanción que le dan un talante federal y que tampoco posee el documento de la ONU. Peculiaridad esta que comporta aspectos tanto positivos como negativos.

Entre los primeros cabe citar sus organizaciones ejecutiva y judicial representadas respectivamente por la Comisión especial formada al efecto y por el Tribunal de Tusticia, instrumentos ambos de gtan perfección técnica en los que se ha hecho gala de notable imaginación para resolver con 


\section{A. ECHANOVE}

equidad las cuestiones delicadas que por definición se deben presentar a ambas instituciones.

La Comisión posee competencia para examinar las quejas presentadas por alguna de las partes contratantes y si éstas lo aceptan incluso por grupos o individuos particulares sobre presuntas violaciones de la Convención. Actuará en consecuencia, en primer lugar, buscando soluciones de conciliación, o en caso negativo, remitiéndose al Consejo de Ministros, y si el Estado afectado lo acepta habrá de ser el Tribunal de Justicia quien decida si ha habido o no tal violación. Todo esto puede suscitar quizás una idea tal vez excesivamente simplificada de la forma en que la Comisión de Derechos Humanos procede. La realidad es harto más compleja y necesitaría de por sí un amplio estudio.

Los resultados prácticos de este aparato jurídico-ideológico no son fáciles de apreciar según datos estadísticos. La experiencia de los años transcurridos bajo la Convención en vigor da que los Estados son más bien reacios a poner en marcha el procedimiento con alguna denuncia, y por lo que a las protestas particulares se refiere, son generalmente de poca monta y o demasiado mal fundamentadas como para promover una requisitoriá. Pero esto mismo indica que las violaciones no son demasiado graves y ostensibles. Por lo demás, la mera existencia de un mecanismo procesal bien articulado acerca de materia tan grave es de gran importan. cia y resulta en sí mismo un mecanismo de disuasión. De ahí se podría licitamente colegir que para valorat con justeza el valor de esta Convención habría que fijarse más en lo que no se ve que en sus actos positivos.

En conclusión, pues, puede decirse que la Convención de Derechos Humanos del Consejo de Europa es, hoy por hoy, el único factor ideológico básico de la construcción europea, junto al cual, el pragmatismo fundamentalmente económico de las Comunidades Europeas se sitúa en un nivel muy inferior.

Junto a este logro hay que contraponer como factor negativo la obligada ineficacia coactiva que a todos los efectos le impone a la Comisión de Derechos Humanos, no sólo la carencia de verdadero poder del Consejo de Europa, sino además un espíritu hecho ya principio, que establece una flexibilidad prácticamente ilimitada para los casos particulares. Con la aplicación excesiva de este espíritu, y es difícil que no se haga así porque coincide con la línea de menor resistencia, toda la eficacia de tan complicado y meritorio organismo puede quedar pura y simplemente volatilizada. 


\section{DESARROLLO DE LA LINEA ECONOMICA}

Resumen: Comienza con la fundación en 1948, de la Organización Curopea de Cooperación toonómica (O.E.C.C.); el paso sigulente, cualitativamente distinto erigen y estructura, es la constituetón de la Comunidad curopea del Carbón y del Acero (C.R.C.A.) en 1951. Continuación y amplación de esta obra son los Tratados de roma, en 195\%, conocidos como IVercado Común o Curopa de los Seis, ampliada a los Nueve en 19\%. Este aconteciniento desencadena, por contraste, dos nuevas organizaciones: la restructuración de la O.E.C.E., que se convierte en la Organización para la Cooperación y el Desarrollo Fconómicos (O.C.D.L.) en 1961, y la creación de la Luropean free Trade Association (E.T.T.A.) o Asociación Europea de Libre Cambio, en 1959.

\section{La O.E.C.E.-O.C.D.E.}

El organismo conocido como Organización Europea de Cooperación Económica fue fundado en 1948 para poner en práctica los acuerdos para la distribución del plan Marshall en los países europeos. Esta finalidad fundacional de la O.E.C.E. queda muy bien expresada en las intenciones del general Marshall, al proponer la ley que lleva su nombre: «No sería bueno, ni útil, que el gobierno de los Estados Unidos emprendiera la tarea de establecer unilateralmente un programa destinado a levantar de nuevo la economía de Europa. Ese es asunto de los europeos. La iniciativa debe venir de Europa.»

Tras este comienzo inserto en causas históricas tan concretas, la trayectoria de la O.E.C.E. ha seguido dependiendo de avatares parecidos, lo que le da un carácter más coyuntural y con menos claridad de objetivos a largo plazo, que los que tienen otras organizaciones internacionales.

La pujanza de la Europa de los Seis, fenómeno completamente ajeno a la O.E.C.E., a partir de 1957, sirvió como catalizador de un proceso de reconversión del organismo, que cobró además nuevas dimensiones al incorporarse a él como miembro, los Estados Unidos que, como es lógico, introdujeron por su misma presencia elementos «deseuropeizadores». Sin embargo, la ampliación del campo condujo a la O.E.C.E. a unos horizontes de utilidad completamente impensados. Perdió la «E» de «Europea» y adquirió la «D» de «Desarrollo». Este cambio de denominación era coherente con una estructura nueva y una finalidad atrayente.

El fin de la nueva organización para el Desarrollo y la Cooperación Económicos (O.C.D.E.) es «promover la ayuda económica y social a través de su área geográfica, asistiendo a los gobiernos miembros en la formulación de políticas orientadas a este fin y cootdinando estas políticas; y estimular y armonizar los esfuerzos de ayuda de sus miembros en favor de los países en vías de desatrollo». 


\section{A. ECHANOVE}

Se consagra desde 1961 al estudio de las politicas econónicas nacio nales y a su interdependencia, constituyendose así en un verdadero centro de investigación, análisis y prospectiva, de extremada utilidad. De ahí, los estudios económicos colectivos gue radografian periódicamente la situación de los Estados miembros-España entre ellos-en función de los planes a largo plazo, y en un sistema de economía de merado, con los problemas que esta conlleva: crecimiento, problemas monetartos, intacion, descmpleo..., etc.

Unicamente hay que recalcar que la O.C.D.E, wi es hoy wa organi. zación estrictamente europea aunge nato como tal, wi puede prescindit de Europa aungue integre paises tan distantes como Japón. Nueva Zelanda o Estados Unidos, pot ejemplo.

\section{Los Tratados de Roma y las Comunidades Europeas}

Muchas son las cosas que se saben sobre las actuales Comunidades Europeas y su organización. No debemos, por tanto, insistir en elenentos tan conocidos por diversas fuentes. En la mente de rodos está el funciom namiento mipolar (Consejo de Ministros Comisión-Parlamento), de la que fue primero la Europa de los Seis, luego de los Nueve y que ahora se enfrenta a la ampliación mediterránea, la más problemática, que la con. vettirá, si se realiza, en la Europa de los Doce.

Tomado en conjunto, el fenóneno de las Comunidades Eutopeas es. sin lugan a dudas, la cota más alta de ambición europeísta. A medida que avanza en sus ampliaciones, a medida que recauda más recursos propios y a medida que van transcurriendo los años en que se ejercitan en el complejo funcionamiento de un auténtico Estado supranacional, las inst. tuciones de la C.E. se consolidan y asientan, haciendose cada vez más imprescindibles, por criticados que sean sus actuates frutos.

Su nacimiento y crecimiento tienen también características peculiates, e incluso simbólicas, desde la thumildad. En 1951 nace el primer núcleo europeo, comuntario e integracionista, la Comunidad Europea del Carbón y del Acero (C.E.C.A.), como parte del proyecto total de reconstucción europen 11 amado «plan Schumant». El fracaso de la C.E.D. en 1954 - ya comentado más arriba-no echó por tierra, sin embargo, el entonces pequeño vástago que era la C.E.C.A. (no obstante integvar dos sectores esenciales de la economía), y ese solo impulso, puramente económico, que suscitaba menos sospechas que la integración politica y militar, sigúó creciendo con fuerza, hasta que sus frutos condujeron a los Acuetdos de Mesina (1955) y Roma (1957), que ampliaron el cuadro de la integración económica europea sobre bases más sólidas aunque más materiales que las nacidas de un prematuro idealismo.

Sin embargo, en las instituciones comuntarias se han manifestado desde entonces dos caracteristicas, no precisamente favorables, que las retatan. 
Una de ellas, aparecida en 1974 con el primer bloqueo petrolifero-ula falta de solidaridad en los momentos dificiles--puso en claro que el realismo de los Tratados de Roma tenía en cuenta solatnente el camino de la prosperidad, y no tanto el de la desgracia.

La otra, muy aireada este mismo año con las elecciones por sutragio universal al Parlanento Europeo, es que los países miembros de la CEE. no han cedido hasta el momento ní un ápice de su soberania nacional.

Si la insolidaridad hace referencia a la úndole psicológica humana y no parece haya de entrar en unas consideraciones como las presentes, la segunda sirve para comprender de un solo golpe el esquema institucional básico del funcionamiento comunitario: una Contión Ejecutiva, gue pro yecta y realiza dentro de las facultades que le son propias; un Pathmento que discute planes y presupuestos, incluso con un derecho de veto que le da apariencia de soberano. Finalmente, un Consejo de Minisiros, representante de los poderes reales, es decir, de los Estados nacionales, que aprueba con benevolencia o veta con frialdad lo que el Parlamento, discursivo, y la Comisión ejecutiva, se afanan por realizar con fuerza relativamente insuficiente.

No hay que subestimar, sin embargo, la ingente labot de las Comunidades. Por mucho que se resistan los Estados a atribuirles poderes, en la Europa de los Nueve existe ya una conciencia común que considera a las Comunidades como un poderoso organismo de unión y unificación.

Por lo tanto, al hablar de las C.E. hay que olvidarse por un monento de la insuficiencia radical que presenta su falta de soberanía, y apreciar, en cambio, sus muchos aspectos positivos.

Es positivo, por ejemplo, respecto del Parlamento europeo, consatar: que su magnitud y la calidad de su representación hayan ido creciendo incesantemente.

Desde los 78 miembros de que constaba la Asamblea Común de la C.E.C.A., se pasó a 142 representantes elegidos con un criterio bísicamente proporcional a la magnitud de los Estados--1o mismo que el Con sejo de Europa-poco después del Tratado de Roma 9 de marzo de 1958), que subieron a 198 cuando en 1974 ingresaron en la Comunidad Gran Bretaña, Irlanda y Dinamarca. El salto más importante, cuantitat va y cualitativamente, se ha producido este mismo año 1979, mediante las elec. ciones del 7 y 10 de junio, por sufragio universal directo, de los 410 dipitados actuales.

La competencia máxima-no la única, naturalmente-de este Parlamento, está en relación con el considerable presupuesto anual de la Comunidad. Téngase en cuenta que actualmente alcanza ya una cifra anual de 12.362 millones de E.C.U. (European Currency Unity), equivalentes a casi dos tercios del presupuesto del Estado Español, la mitad del belga y un octavo del de Francia. 


\section{A. ECHANOVE}

En el orden puramente político es difícil saber lo que a partir de ahora puede esperarse. Pero la nueva índole representativa del actual Patamento permite augurax, cuando menos, una actividad pensante nutrida, contrastada y enriquecida por todas las tendencias politicas allí presentes, lo cual alcanzará sin duda los límites teóricos de lo posible en el camino de la integración. Si el cambio de mentalidad en orden a una integración europea puede operarse, será el nuevo Parlamento quien lo consiga. En palabras de Roy Jenkins, presidente de la Comisión, la elección del nuevo Parlamento se acompaña de posibilidades nuevas: «las que le procuran la autoridad moral y la influencia acrecidas de que goza. La cuestión de los poderes del Parlamento europeo recién elegido no es pues mi preocupación primaria. Gracias a su autoridad moral acrecentada, la nueva asamblea tendrá muchas ocasiones de ejercer una influencia creciente con los poderes que actualmente tiene».

Entre tanto, la Comisión, el órgano más efectivo y estructuralmente más articulado de las instituciones comunitarias, mantiene y acrecienta su capacidad de acción como «gobierno europeo». A la Comisión pertenece la triple competencia que le confiere su función fundamental de motor de la Comunidad, a saber:

a) La capacidad de iniciativa en virtud de la cual es el único órgano que de derecho puede elaborar y presentar las propuestas de todos los actos de la Comunidad y someterlos a los poderes decisorios, es decir, al Consejo de Ministros.

b) La vigilancia del cumplimiento de los tratados, especialmente por lo que toca a los defectos que puedan observarse de parte de cada uno de los Estados miembros.

c) Competencia exclusiva de gestión y ejecución de las reglas comunes, con relativamente amplios poderes de decisión propia.

Sería inacabable, y más propio de una pequeña historia del Mercado Común, pretender reflejar en datos concretos la actividad de la Comisión. Unicamente y por lo que tiene de reflejos institucionales, diremos que la distribución del trabajo está encomendada a 13 comisarios, incluido el Presidente y cuatro Vicepresidentes, que aunque trabajan colegialmente, se responsabilizan individualmente de áreas espcifficas.

Junto a este trípode institucional que forman la Comisión y el Par. lamento, con el Consejo de Ministros, el cuarto poder, o autoridad judicial, tiene el papel y competencias que pueden deducirse, representado por un Tribunal de Justicia, residente en Estrasburgo y en todo acomodado, lógicamente, a la estructura y complejidad de los demás estamentos básicos.

Para terminar, es obvio que digamos que existe una autoridad incluso superior a la del Consejo de Ministros, formada por el llamado Consejo Europeo, de los jefes de los Estados miembros, que se reúne eventual- 
mente cuando lo cree preciso, para la adopción de medidas o iniciativas de mayor envergadura. Las últimas de estas reuniones en la cumbre, en Bremen y Bonn, durante el año pasado, sirvieron para poner en maxcha la importantísima reforma del sistema monetario, del que el mencionado y famoso E.C.U., un poco precipitadamente llamado moneda europea, es sólo una parte.

\section{La EFTA (European Free Trade Association)}

Habiendo aludido más arriba a la transformación realizada en la O.E.C.E. como consecuencia, en parte, del nuevo fenómeno económico representado por la Europa de los Seis, nos queda finalmente por decir una palabra acerca de la última institución aparecida en la dinámica europea.

La Asociación Europea de Libre Cambio o EFTA, no es una asociación de auténtica iniciativa sino más bien una resultante defensiva ante la verdadera iniciativa que constituían los diversos tratados del Mercado Común.

En 1959, recuérdese que dos años antes habían sido definitivamente firmados los Tratados de Roma, se empezó a forjat la necesidad de formar un organismo paralelo que tuviera como objeto exclusivo la liberalización del comercio de bienes industriales y agrícolas entre todos los países que cubrían el área geográfica de la O.C.D.E. Se pensaba en los ya agluinados en el Mercado Común (los Seis) como una determinada unidad que liberalizara a su vez todos los cambios de sus países respecto de todos los demás incluidos en la O.C.D.E., lo cual significaría en la práctica un inunto de aguar la eficacia del Mercado Común. Naturalmente, éste no aceptó tal base y continuó su propia ruta mientras los países agrupados en la EFTA y acaudillados por Gran Bretaña emprendían un camino de pura liberalización comercial para de alguna manera no quedarse atrás frente al despegue económico que manifestaban sus oponentes.

La estructura de la EFTA difería fuertemente de la del Mercado Común. Su mismo principio de no coacción, esencial al librecambismo, hacía que cada país actuara con personalidad independiente respecto de cualquier comercio exterior a la EFTA, mientras se coordinaba con los demás países que componían la asociación mediante un patrón de reducción de tarifas arancelarias o supresión de ellas. Esto era un arma de dos filos, porque si bien dejaba a los países miembros una capacidad de iniciativa comercial ilimitada, creaba una red de facilidades diversas hacia el exterior por la cual cada producto procedente de fuera del área podría encontrar en alguno de los países miembros el agujero de penetración que considerara más favorable. Si al Mercado Común se le podía entonces y se le puede todavía echar en cara cierta ineficacia de sus resortes de supranacionalidad, en el caso de la EFTA, cualquiet atisbo de supranacionalidad eva por principio impensable. En este sentido, el Mercado Común de los Tratados de Roma siempre llevaría ventaja, como se ha manifestado 


\section{A. ECHANOVE}

en todo su volumen de cambios comerciales y en la significación de conjunto que ha adquirido.

La prineta crisis grave de la EFTA sobrevino en 1963, tan sólo tres años despues de que la Convención fundacional de la EFTA (1960) hubiexa conenzado a ser operativa, cuando Gran Bretaña solicitó unilateralmene la entrada en el Mercado Común, desamparando a los demás países de la Asociación. Pot entonces el general De Gaulle frustró tal intento con su portazo a Inglaterta, y las cosas volvieron a st cauce, pero años más tarde, er 1972, Gran Bretana y Dinamara, ambos pertenecientes a la EFIA, solicitaron su ingreso en el Mercado Común, y la EFTA se véa bligada a realizar un nuevo esfuerzo de reajuste para seguir manteniendo las litritadas ventajas de su liberalización comercial nutua.

Hoy dia la EFTA se compone de siete países (Austria, Suiza, Suecia, Noruega, Portugal, Finlandia, Islandia) a los que nos acabamos de unir el día 26 de junio los propios espanoles en espera de la admisión definitiva, cuando ésta se produzca, en el Mercado Común.

\section{Conclustón}

Si se recapitula todo el armazón anteriormente expuesto, pueden extraerse algunas consecuencias que apenas implican juicio de valoz.

1. Por diferentes que aparezcan las denominaciones, el carácter (cooperativo integtacionista), las finalidades especificas y las estructuras organizativas, todos estos movinientos están entre sí vinculados por su origen histórico, por área geográfica u otras afinidades. Por tanto, el alcance y la finalidad de cada uno difícilmente pueden calibrarse sin tener en cuenta el contexto global, o lo que podría denominarse «clima anitario».

2. La complementariedad o la suplementariedad son muy frecuentes entre ellos, o porque la cobertura de países sea absolutamente diferente (caso de la CEE y de la EFT.A) o porque la finalidad específica de cada organismo permite a cada país pertenecer a varios simultáneamente sin incurrir en políticas contradictorias.

Cabe preguntarse si todos ellos son igualmente necesarios, to que podra responderse de diversa forma. Pero en general puede decirse que cada uno ha ido ocupando su propia parcela o por acierto original o por adaptación a nuevas funciones, como es el caso de la OECE-OCDE.

3. Aunque cada organización internacional europea reclama sa propia autonomía, esta pretensión es inoperante en la mayoría de los casos. Todas ellas tienen una radical y marcada dependencia, fundamentalmente de los gobtemos nacionales de los Estados miembros, que poseen toda la capacidad de decisión politica, y de las aportaciones econónicas para su sostenimiento. De esta condición sólo empiezan a emanciparse las Comunidades Europeas, debido a los tributos propios que por fin exacciona. Así 
y todo, ni la Comisión ni el Parlamento pueden nover un dedo para la transformación política contra la voluntad de los gobiemos nacionales re. presentados por el Consejo de Ministros. Y ouro tano le ocure al Consejo de Europa.

4. A esta dependencia hay que sumar la que sobre todo en el terreno militar, pero también en el económico, existe respecto de los Estados Unidos. Percibir esta fudamental debilidad es tocar con el dedo la yerda dera situación de la derotada Europa, y a ella no escapa tampoco la pretension autonomista de Francia.

5. Aun con estas limitaciones y dentro del estrecho margen de maniobra que conllevan, las organizaciones europeas están tealizando un increible trabajo, materializado en una tupidísima red de convenios, consulas y estudios. Si a pesar de ellos no se ha conseguido aún la finalidad que los padres de Europa parecía iban a ver con sus ojos, ello no hace otra cosa que patentizar las excesivas desigualdades históricas que existen bajo la denominacion «Europa». Para que se opere el adecuado cambio de mentalidad es paso previo el llegar mediante acuetdos cooperativos o integracionistas de menor ambición a homologaciones jurúlicas, económicas, politicas y militares, y a sistemas parciales de compensación. (Europa verde, desigualdades regionales) que entrañan complejas dificultades técnicas y superación de prejuicios o recelos inveterados.

6. Los términos en que se ha planteado la crisis energética y económica - acentuada en los últimos mesesmponen un nuevo paréntesis a todo al ritmo de desarrollo auropeísta. No hay que excluir la posibilidad de que precisamente en una adversidad más permanente que coyuntural encuentre Europa la imaginativa necesaria para impulsar decidida y rápidamente toda la uniticación posible. Deliberadamente rehusamos profundizar, por aiena a la indole del artículo, en la filosofía práctica y modificación de valores que tal situación presupone. 